\title{
Transcranial direct current stimulation for promoting motor function in cerebral palsy: a review
}

\author{
Melanie K. Fleming ${ }^{1 *}$ (D) Tim Theologis ${ }^{2}$, Rachel Buckingham ${ }^{2}$ and Heidi Johansen-Berg ${ }^{1}$
}

\begin{abstract}
Transcranial direct current stimulation (tDCS) has the potential to improve motor function in a range of neurological conditions, including Cerebral Palsy (CP). Although there have been many studies assessing tDCS in adult stroke, the literature regarding the efficacy of tDCS in CP is more limited. This review therefore focuses on the neurophysiological and clinical findings in children and adolescents with CP. Initial studies applying anodal tDCS to promote lower limb function are promising, with improvements in gait, mobility and balance reported. However, the results of upper limb studies are mixed and more research is needed. Studies investigating neurophysiological changes or predictors of response are also lacking. Large-scale longitudinal studies are needed for the lower limb to ascertain whether the initial pilot results translate into clinically meaningful improvements. Future studies of the upper limb should focus on determining the optimal stimulation parameters and consider tailoring stimulation to the individual based on the (re)organisation of their motor system.
\end{abstract}

Keywords: Cerebral palsy, Motor function, Transcranial direct current stimulation, Brain stimulation, Upper limb, Lower limb

\section{Introduction}

Transcranial direct current stimulation (tDCS), a form of non-invasive brain stimulation, has received considerable interest as a neuromodulatory technique with the potential to enhance cortical plasticity and improve motor function in a range of neurological conditions. Low intensity, direct, constant current is applied to the scalp (Fig. 1), typically over the primary motor cortex (M1), and cortical excitability and inhibition is altered depending on the stimulation parameters $[1,2]$ (Table 1).

One of the neurological conditions which may benefit from the neuromodulatory effects of tDCS is Cerebral Palsy $(\mathrm{CP})$, whereby motor function and development are affected by an insult to the developing brain [3]. Since functional deficits limit independence and quality of life long term, the potential to utilise tDCS as an adjunct to physical therapy for enhancing motor function

\footnotetext{
* Correspondence: melanie.fleming@ndcn.ox.ac.uk

${ }^{1}$ Wellcome Centre for Integrative Neuroimaging, FMRIB, Nuffield Department of Clinical Neurosciences, University of Oxford, John Radcliffe Hospital, Oxford OX3 9DU, UK

Full list of author information is available at the end of the article
}

is an attractive concept. However, although there has been considerable investigation of the effectiveness of tDCS for adult stroke, the findings cannot be assumed to translate directly into children and adolescents with $\mathrm{CP}$, due to differences in brain size, maturity, anatomy and reorganisation. The application of tDCS in this population appears to be safe [4] and safety guidelines have been developed [5]. This narrative review therefore focuses on the neurophysiological and clinical findings with use of tDCS in children and adolescents (6-21 years) with $\mathrm{CP}$.

\section{Information sources}

References for this review were identified, by MF, through searches of PubMed for articles published up to July 2018. Combinations of the terms "cerebral palsy", "tdcs", "brain stimulation", "child stroke" and "pediatric stroke" were used. Additionally, articles were identified through article reference lists. The final reference list was selected, by MF, on the basis of topic relevance.

(C) The Author(s). 2018 Open Access This article is distributed under the terms of the Creative Commons Attribution 4.0 International License (http://creativecommons.org/licenses/by/4.0/), which permits unrestricted use, distribution, and reproduction in any medium, provided you give appropriate credit to the original author(s) and the source, provide a link to the Creative Commons license, and indicate if changes were made. The Creative Commons Public Domain Dedication waiver (http://creativecommons.org/publicdomain/zero/1.0/) applies to the data made available in this article, unless otherwise stated. 

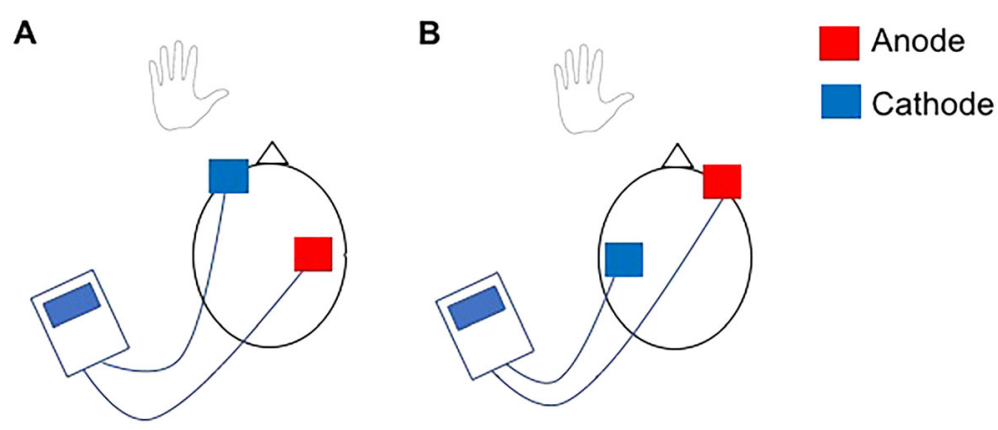

Fig. 1 Diagrammatic representation of tDCS. a Anodal stimulation applied over the motor cortex contralateral to the trained limb. $\mathbf{b}$ Cathodal stimulation applied over the motor cortex ipsilateral to the trained limb, based on the interhemispheric imbalance model

\section{Neurophysiological findings}

Assessment of change in cortical activity or excitability is important in order to understand the mechanism of action of tDCS. Additionally, differences in neurophysiological outcomes may potentially be of use to explain variability in clinical outcome, while variations in neurophysiological measures at baseline may be able to predict who will benefit from tDCS. Currently, there are very few studies in CP which have reported using brain imaging or neurophysiological measures alongside tDCS.

TDCS is known to alter cortical excitability, intracortical inhibition, and cortical plasticity [1, 2, 6, 7] and these neuromodulatory effects are thought to underlie the behavioural or clinical efficacy of tDCS. Transcranial Magnetic Stimulation (TMS) is commonly used to assess changes in cortical excitability or intracortical inhibition following a single session of tDCS in adult stroke $[8,9]$. However, to our knowledge, there are no published studies of this type in $\mathrm{CP}$. One study [10] reported an increase in motor evoked potential (MEP) amplitude elicited by TMS following 10 days of anodal tDCS ( $1 \mathrm{~mA}, 20 \mathrm{~min})$ targeting the lower limb. MEPs were elicited at $110 \%$ rest motor threshold (RMT) from the abductor muscle of the thumb and the quadriceps muscle of the lower limb at rest. Each hemisphere was stimulated separately, but the results do not separate the findings from

Table 1 Fundamentals of tDCS

\footnotetext{
- Typically, two electrodes are placed on the scalp, one over the area of interest (e.g. motor cortex), and current flows between them

- Typical sensations include tingling, prickling and itching of the scalp

as the current intensity ramps up

- Sham stimulation can be effectively applied by ramping up

stimulation for a short period, then turning it off

- Current direction, duration and intensity all require consideration

- Anodal tDCS typically enhances cortical excitability and reduces

inhibition, cathodal tDCS typically suppresses excitability

- tDCS can be delivered at rest or during a task, e.g. motor training

- Effects can be seen during and after tDCS

- Single session effects can be seen, or cumulative effects over multiple sessions
}

each muscle or hemisphere. Therefore, although anodal tDCS appeared to increase cortical excitability, as hypothesised, it is unclear as to how specific the changes are to the targeted region or the time-scale over which these changes occurred.

Changes in brain metabolites following tDCS can be assessed using Magnetic Resonance Spectroscopy (MRS) $[2,11,12]$. This can provide insights into alterations in measures of neuronal health or changes in levels of cortical inhibitory or excitatory neurotransmitters. Auvichayapat et al. [13] attempted to assess changes in brain metabolites following tDCS using MRS in children with CP. Anodal tDCS $(20 \mathrm{~min}, 1 \mathrm{~mA})$ was delivered for 5 consecutive days to the left M1 in children 8-12 years old with spastic $\mathrm{CP}$ affecting their right upper limb. They reported a significant increase in concentrations of $\mathrm{N}$-acetylaspartate (NAA), Choline and Myoinositol in the left basal ganglia and an increase in the ratio of Glx (a combination of glutamate and glutamine) to Creatine in the left M1. Although there was no sham control group, the authors speculated that the tDCS-induced increase in activity of the M1 leads to an increase in the concentration of NAA, Choline and Myoinositol in the basal ganglia. There was a negative correlation between the ratio of Glx:Creatine in the M1 and the spasticity (Tardieu scale score) of the right upper limb (shoulder flexors, shoulder external rotators, elbow flexors and elbow pronators) following tDCS. However, the authors did not report whether this relationship existed at baseline or whether the change in metabolite ratios correlated with change in spasticity. There was also no indication of the quality of the MRS data, which is typically an important consideration in MRS studies. High quality MRS data may be difficult to obtain in this population, especially in regions such as the basal ganglia.

\section{Upper limb function}

Research on the effect of tDCS on upper limb function in $\mathrm{CP}$ is limited to date (Table 2). Similar to adult stroke [14] the studies that have been published have utilised the 


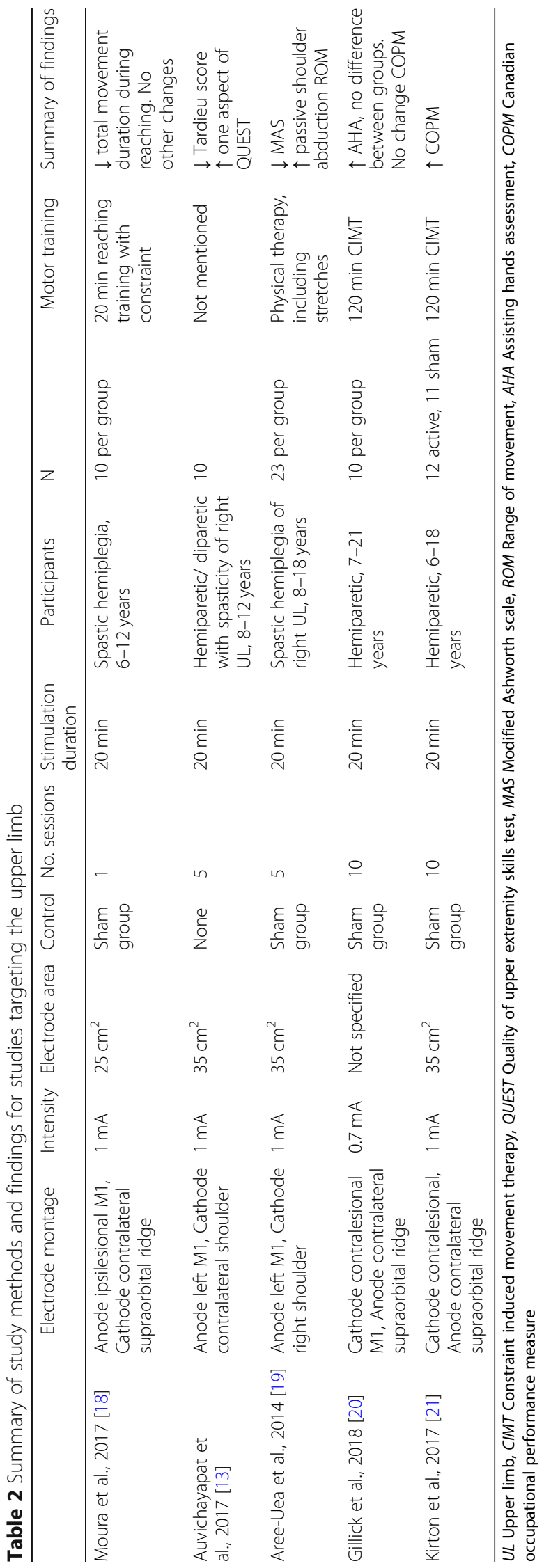


"interhemispheric imbalance model" as rationale. The interhemispheric imbalance model proposes that there are abnormal levels of interhemispheric inhibition from the contralesional to ipsilesional M1, resulting in a reduction in activity of the ipsilesional M1 during movement of the affected limb and an increase in activity of the contralesional M1 [15-17]. Therefore, this model provides rationale for applying anodal tDCS to the ipsilesional M1 to increase excitability, or cathodal tDCS to the contralesional M1 in an attempt to decrease excitability and thereby upregulate the ispilesional M1 through a reduction in interhemispheric inhibition from the contralesional hemisphere.

A single session study [18] delivered $20 \mathrm{~min}$ of $1 \mathrm{~mA}$ anodal tDCS (or sham) to the ipsilesional M1 of children with spastic hemiplegia, alongside $20 \mathrm{~min}$ of motor training of the affected arm with constraint of the other arm. Using motion analysis, a significant reduction in total movement duration during reaching movements with the affected hand was observed for the tDCS group compared with sham. Although this initially seems promising, there were numerous comparisons made, and none of the other change values (e.g. smoothness, velocity or accuracy parameters) showed significant between-group differences.

Two studies have delivered multiple sessions of anodal tDCS in CP [13, 19]. Auvichayapat et al. [13] delivered 5 days of tDCS to the left M1. Although there was a mixture of hemiparetic and diparetic participants, all had spasticity of their right upper limb. However, there is no mention as to whether there was any motor training alongside the tDCS. Although the authors reported an improvement in spasticity (Tardieu scale) and one aspect of the Quality of Upper Extremity Skills Test (QUEST), there was no sham group for comparison. A randomised, double-blinded study [19] aimed to assess changes in spasticity with 5 consecutive days of anodal tDCS (20 min, $1 \mathrm{~mA}$ ) to the left M1 of children with spastic hemiplegia affecting the right arm. In addition to the tDCS, participants engaged in "routine physical therapy", including passive and active stretching, therapeutic positioning and aerobic exercise. There were improvements in spasticity of the shoulder, elbow, wrist and fingers and an improvement in shoulder abduction passive range of movement for the active tDCS group only. However, there were no active motion function measures assessed.

Two double-blind randomised trials $[20,21]$ have combined 20 min of cathodal tDCS of the contralesional M1 with motor training, including constraint induced movement therapy (CIMT), over 10 sessions in children with hemiparetic $\mathrm{CP}$. Both active and sham groups demonstrated a significant increase in the Assisting Hand Assessment (AHA), which measures bimanual function during novel play or functional tasks, but there was no difference between groups. Kirton et al. [21] did find greater improvement in self-reported performance (using the Canadian Occupational Performance Measure (COPM)) for the active tDCS group, and a higher proportion of participants achieved a clinically significant improvement on this measure compared to the sham group. However, the COPM did not show between-group differences in the study by Gillick et al. [20], indicating that more research is needed with both objective and subjective measures.

The intensity of the current for cathodal tDCS may be an issue in the studies so far. Contrary to effects in adults [1], in a study with healthy children (11-16 years) [22], corticospinal excitability was found to increase, rather than decrease, following $1 \mathrm{~mA}$ cathodal tDCS. If the intensity of stimulation was lowered to $0.5 \mathrm{~mA}$ then the hypothesised decrease in MEP amplitude for cathodal tDCS was evident. Moliadze et al. therefore speculated that $0.5 \mathrm{~mA}$ cathodal stimulation in children may produce similar effects as $1 \mathrm{~mA}$ in adults. The situation is different from anodal stimulation: $0.5 \mathrm{~mA}$ anodal stimulation was found to be ineffective at increasing MEP amplitude in children whereas $1 \mathrm{~mA}$ anodal stimulation did lead to a significant increase [22], consistent with effects of anodal tDCS in adults [1].

Therefore, there is currently no indication that tDCS provides additional benefit for active motor function over motor training or CIMT alone in children and young people with $\mathrm{CP}$, but spasticity appears to improve with anodal tDCS.

\section{Lower limb function}

A summary of studies delivering tDCS to target lower limb function is provided in Table 3. To our knowledge, there are only two single-session studies of tDCS in CP targeting lower limb function [23, 24]. One study [23] delivered $20 \mathrm{~min}$ of anodal tDCS $(1 \mathrm{~mA})$ at rest to the dominant hemisphere in participants with hemiparetic or diparetic CP. Motion analysis was used to assess balance and gait before, immediately following and $20 \mathrm{~min}$ later. There was a significant reduction in sway and an increase in walking speed for the active group compared with sham, but no change in cadence. However, the results of the study by Lazzari et al. [24] are less promising. Anodal tDCS was delivered to the motor cortex (the authors do not specify which hemisphere) for $20 \mathrm{~min}$ (1 $\mathrm{mA}$ ) in combination with $20 \mathrm{~min}$ of mobility training using virtual reality (Xbox 360 with Kinect movement sensor (Microsoft Corporation, Redmond, WA)). The virtual reality training involved walking with and without simulated obstacles. Static balance was assessed using a force plate. The authors report an increase in sway velocity immediately following the intervention for both groups, but no clear between-group differences. There was no later follow-up assessment and therefore the 


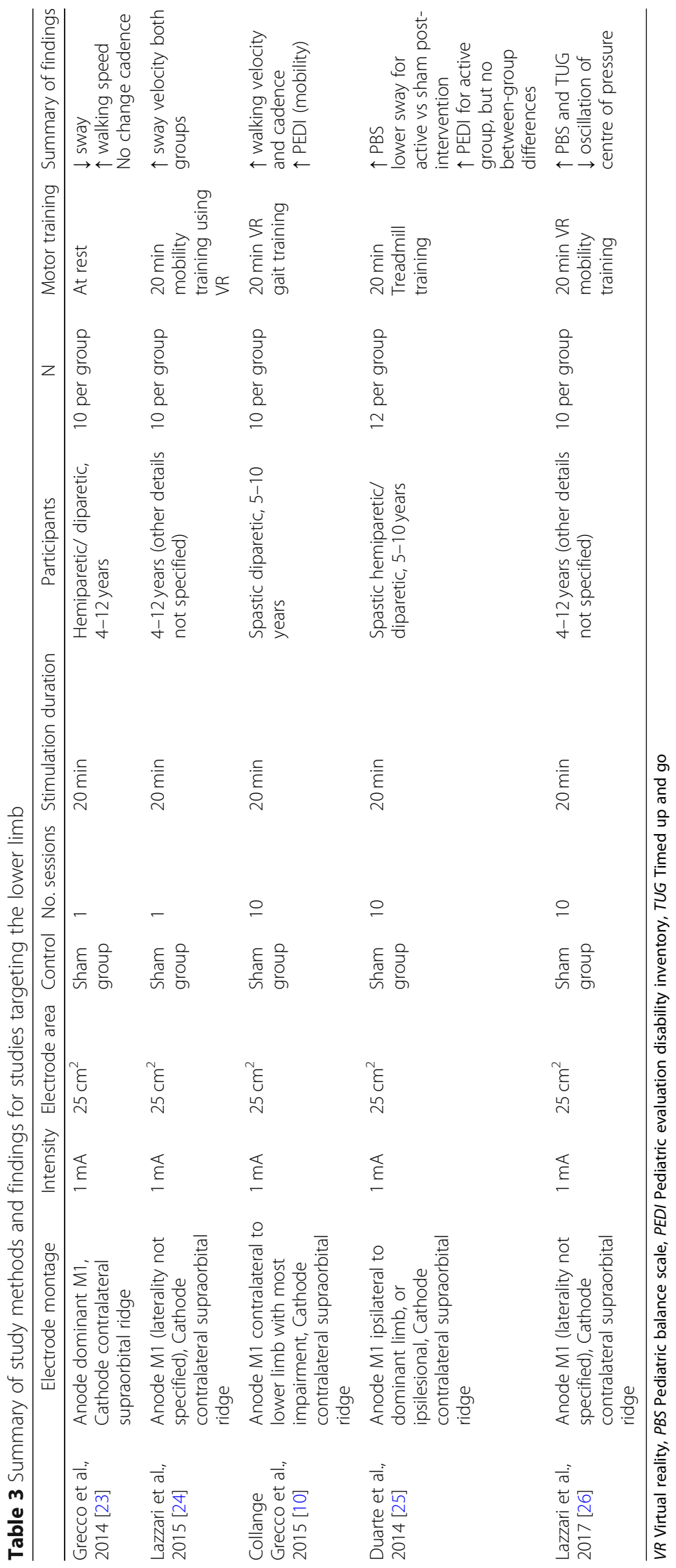


increase in sway velocity observed immediately could represent a deterioration in balance due to fatigue for both groups following the mobility training. If this is the case, then it would appear that tDCS was not effective at ameliorating this fatigue effect.

Three studies have assessed multiple sessions of anodal tDCS for promoting lower limb function $[10,25,26]$. Duarte et al. [25] delivered 10 sessions of anodal tDCS (1 $\mathrm{mA}, 20 \mathrm{~min}$ ), in combination with treadmill training in children with spastic CP. There was a mixture of hemiparetic and diparetic participants and the anode was placed over the motor cortex ipsilateral to the dominant limb (thereby stimulating the more-affected hemisphere). Interpretation is complicated as the authors report within group changes and between group score comparisons separately, rather than using a mixed analysis of variance or change scores. Nonetheless, within-group comparisons demonstrated an improvement in the Pediatric Balance Scale (PBS) for the active tDCS group only, and between-group comparisons showed that the active group had a higher PBS score and lower sway than the sham group when assessed following the intervention and at the 1 month follow-up. Similarly, there was an improvement for the active group on the mobility subsection of the Pediatric Evaluation Disability Inventory (PEDI), which is a subjective assessment of functional performance in activities of daily living. However, the scores did not differ between groups.

Collange Grecco et al. [10] used virtual reality for gait training in combination with 10 sessions of anodal tDCS $(1 \mathrm{~mA}, 20 \mathrm{~min})$ in children with spastic diparetic $\mathrm{CP}$. The virtual reality training involved walking around a simulated race track at varying speeds (Xbox 360 with Kinect movement sensor (Microsoft Corporation, Redmond, WA)). Participants were asked which lower limb they found had most difficulty during gait and the anode was placed over the contralateral motor cortex. Their primary outcome measure was gait kinematics, using motion analysis. There was a greater improvement in walking velocity and cadence for the tDCS group compared to sham, but not for any of the other gait variables assessed. Mobility, assessed using the PEDI, also improved for the active tDCS group, but not for sham. Similarly promising results were found by Lazzari et al. [26], who combined anodal tDCS (1 mA, $20 \mathrm{~min}$ ) with $20 \mathrm{~min}$ of mobility training using virtual reality over 10 sessions. The virtual reality training involved a game that simulates stationary walking requiring complete flexion of the hip, knee and ankle, and weight transfer from one limb to the other (Xbox 360 with Kinect movement sensor (Microsoft Corporation, Redmond, WA)). They demonstrated a significantly greater improvement in the PBS and the Timed Up and Go (TUG) for the active tDCS group compared with sham. There was also a greater improvement in static balance, assessed as the oscillation of the centre of pressure. However, variability within groups was high and there was no investigation of variables to account for variability.

\section{Predictors of response}

Data on the predictors of response to tDCS are currently lacking in this population. The only study to attempt to analyse potential predictors [27] did so by combining 3 studies that delivered anodal tDCS alongside gait training (for a total of 56 participants) in children with spastic hemiparetic or diparetic CP. The authors reported that two predictors were significantly associated with the responsiveness to the intervention; MEP presence during initial evaluation (indicating preservation of the corticospinal tract) and location of the injury (cortical or subcortical). However, it is unclear whether this is specific to the modulatory effects of the tDCS per se or simply an indicator of who has the potential to improve motor function, as is the case for adult stroke survivors [28].

For the upper limb, it is currently unclear whether anodal or cathodal tDCS should be applied in unilateral CP. Indeed, this decision may depend on the extent to which the activity of each M1 is required for motor function, based on the degree to which the ipsilesional M1 and corticospinal tract are damaged. Although in some instances, over-activity of the contralesional hemisphere may be maladaptive [29] and benefit from downregulation, in other cases the motor system may be reorganised towards ipsilateral control [30, 31]. If the control of the paretic hand is through fast-conducting ipsilateral projections from the contralesional M1, then attempting to enhance ipsilesional M1 excitability with anodal tDCS may be futile. Equally, decreasing excitability of the contralesional M1 with cathodal tDCS might be detrimental, as is seen for people with severe upper limb impairment after adult stroke [32]. It is difficult to determine whether someone with $\mathrm{CP}$ relies on ipsilateral control from the contralesional hemisphere based on clinical presentation alone, as children with ipsilateral projections can show a useful grasp, or no movement at all [30]. Therefore, measures, such as Diffusion Tensor Imaging (DTI) to assess fractional anisotropy of the corticospinal tract, or TMS to assess corticospinal tract integrity through the presence or absence of MEPs, may be necessary for informing choices with regard to electrode placement. We therefore propose that future studies attempt to optimise tDCS delivery, based on knowledge of the (re)organisation of the individual's motor system.

There is currently no investigation into the optimal age for delivery of tDCS. The studies presented here have delivered tDCS to children and adolescents, but it is conceivable that the responsiveness could be dependent on the stage of development of the individual. Therefore, future studies are needed to address this issue. 


\section{Conclusions}

Application of tDCS for enhancing lower limb function in young people with $\mathrm{CP}$ appears effective, although largescale longitudinal studies are required to confirm the initially promising findings. Further single-session and longitudinal studies are required to determine the efficacy of tDCS for the upper limb and to elucidate mechanisms of action and predictors of response in this population.

\section{Abbreviations}

AHA: Assisting Hand Assessment; CIMT: Constraint induced movement therapy; COPM: Canadian Occupational Performance Measure; CP: Cerebral Palsy; DTI: Diffusion Tensor Imaging; M1: Primary motor cortex; MEP: Motor evoked potential; MRS: Magnetic Resonance Spectroscopy; NAA: N-acetylaspartate; PBS: Pediatric Balance Scale; PEDI: Pediatric Evaluation Disability Inventory; QUEST: Quality of Upper Extremity Skills Test; RMT: Rest motor threshold; tDCS: Transcranial direct current stimulation; TMS: Transcranial magnetic stimulation; TUG: Timed Up and Go

\section{Acknowledgements}

Not applicable.

\section{Funding}

H.J.B. holds a Wellcome Principal Research Fellowship (Grant 110027/Z/15/Z). The Wellcome Centre for Integrative Neuroimaging is supported by core funding from the Wellcome Trust (Grant 203139/Z/16/Z). The funders had no role in the writing of the manuscript.

\section{Availability of data and materials}

Not applicable.

\section{Authors' contributions}

MKF reviewed the articles and was the major contributor to the article. HJB, $\Pi$ and RB contributed to the basic concept of the paper and criticially revised the draft paper. All authors read and approved the manuscript.

\section{Ethics approval and consent to participate}

Not applicable.

\section{Consent for publication}

Not applicable.

\section{Competing interests}

The authors declare that they have no competing interests.

\section{Publisher's Note}

Springer Nature remains neutral with regard to jurisdictional claims in published maps and institutional affiliations.

\section{Author details}

${ }^{1}$ Wellcome Centre for Integrative Neuroimaging, FMRIB, Nuffield Department of Clinical Neurosciences, University of Oxford, John Radcliffe Hospital, Oxford OX3 9DU, UK. ${ }^{2}$ Nuffield Orthopaedic Centre, Oxford University Hospitals NHS Foundation Trust, Oxford, UK.

\section{Received: 30 August 2018 Accepted: 14 December 2018}

Published online: 20 December 2018

\section{References}

1. Nitsche M, Paulus W. Excitability changes induced in the human motor cortex by weak transcranial direct current stimulation. J Physiol. 2000;527(Pt 3):633-9 Available from: http://www.ncbi.n/m.nih.gov/pubmed/10990547.

2. Stagg CJ, Best JG, Stephenson MC, O'Shea J, Wylezinska M, Kincses ZT, et al. Polarity-sensitive modulation of cortical neurotransmitters by transcranial stimulation. J Neurosci. 2009;29:5202-6 Available from: http://www.jneurosci. org/cgi/doi/10.1523/JNEUROSCI.4432-08.2009.

3. Rosenbaum P, Paneth $N$, Leviton A, Goldstein M, Bax M, Damiano D, et al. A report: The definition and classification of cerebral palsy April 2006. Dev
Med child Neurol Suppl. 2007;109:8-14 Available from: https://www.ncbi. nlm.nih.gov/pubmed/17370477.

4. Krishnan C, Santos L, Peterson MD, Ehinger M. Safety of noninvasive brain stimulation in children and adolescents. Brain Stimul. 2016;8:76-87 Available from: https://www.ncbi.n/m.nih.gov/pubmed/25499471.

5. Gillick BT, Gordon AM, Feyma T, Krach LE, Carmel J, Rich TL, et al. Noninvasive brain stimulation in children with unilateral cerebral palsy: a protocol and risk mitigation guide. Front Pediatr. 2018;6:1-9 Available from: http://journal.frontiersin.org/article/10.3389/fped.2018.00056/full.

6. Kidgell DJ, Daly RM, Young K, Lum J, Tooley G, Jaberzadeh S, et al. Different current intensities of anodal transcranial direct current stimulation do not differentially modulate motor cortex plasticity. Neural Plast. 2013;2013:13-5 Available from: https://www.ncbi.nlm.nih.gov/pubmed/23577272.

7. Huang YZ, Lu MK, Antal A, Classen J, Nitsche M, Ziemann U, et al. Plasticity induced by non-invasive transcranial brain stimulation: a position paper. Clin Neurophysiol. 2017;128:2318-29. https://doi.org/10.1016/j.clinph.2017. 09.007 International Federation of Clinical Neurophysiology.

8. Bastani A, Jaberzadeh S. Does anodal transcranial direct current stimulation enhance excitability of the motor cortex and motor function in healthy individuals and subjects with stroke: a systematic review and meta-analysis. Clin Neurophysiol. 2012;123:644-57. https://doi.org/10.1016/j.clinph.2011.08. 029 International Federation of Clinical Neurophysiology.

9. Fleming MK, Pavlou M, Newham DJ, Sztriha L, Teo JT. Non-invasive brain stimulation for the lower limb after stroke: what do we know so far and what should we be doing next? Disabil Rehabil. 2017;39:714-20 Available from: https://www.ncbi.n/m.nih.gov/pubmed/27013330.

10. Collange Grecco LA, De Almeida Carvalho Duarte N, Mendonça ME, Galli M, Fregni F, Oliveira CS. Effects of anodal transcranial direct current stimulation combined with virtual reality for improving gait in children with spastic diparetic cerebral palsy: a pilot, randomized, controlled, double-blind, clinical trial. Clin Rehabil. 2015;29:1212-23 Available from: https://www.ncbi. nlm.nih.gov/pubmed/25604912.

11. Bachtiar $V$, Johnstone $A$, Berrington A, Lemke $C$, Johansen-Berg $H$, Emir $U$, et al. Modulating regional motor cortical excitability with non-invasive brain stimulation results in neurochemical changes in bilateral motor cortices. J Neurosci. 2018;38:2853-17 Available from: http://www.jneurosci.org/lookup/ doi/10.1523/JNEUROSCI.2853-17.2018.

12. Bachtiar V, Near J, Johansen-Berg H, Stagg CJ. Modulation of GABA and resting state functional connectivity by transcranial direct current stimulation. Elife. 2015;4:1-9 Available from: https://www.ncbi.nlm.nih.gov/ pubmed/26381352.

13. Auvichayapat $P$, Aree-uea B, Auvichayapat N, Phuttharak W, Janyacharoen T, Tunkamnerdthai $\mathrm{O}$, et al. Transient changes in brain metabolites after transcranial direct current stimulation in spastic cerebral palsy: a pilot study. Front Neurol. 2017;8:1-9 Available from: http://journal.frontiersin.org/article/ 10.3389/fneur.2017.00366/full.

14. Ward NS, Cohen LG. Mechanisms underlying recovery of motor function after stroke. Arch Neurol. 2004;61:1844-8 Available from: https://www.ncbi. nlm.nih.gov/pubmed/15596603.

15. Murase N, Duque J, Mazzocchio R, Cohen LG. Influence of interhemispheric interactions on motor function in chronic stroke. Ann Neurol. 2004;55:400-9.

16. Takeuchi N, Tada T, Toshima M, Ikoma K. Correlation of motor function with transcallosal and intracortical inhibition after stroke. J Rehabil Med. 2010;42: 962-6.

17. Takeuchi N, Izumi S-I. Noninvasive brain stimulation for motor recovery after stroke: mechanisms and future views. Stroke Res Treat. 2012;2012:1-10 Available from: http://www.hindawi.com/journals/srt/2012/584727/.

18. Moura RCF, Santos C, Collange Grecco L, Albertini G, Cimolin V, Galli M, et al. Effects of a single session of transcranial direct current stimulation on upper limb movements in children with cerebral palsy: a randomized, sham-controlled study. Dev Neurorehabil. 2017;20:368-75 Available from: https://www.ncbi.nlm.nih.gov/pubmed/28632467.

19. Aree-Uea B, Auvichayapat N, Janyacharoen T, Siritaratiwat W, Amatachaya A, Prasertnoo J, et al. Reduction of spasticity in cerebral palsy by anodal transcranial direct current stimulation. J Med Assoc Thail. 2014;97(9):954-62.

20. Gillick B, Rich T, Nemanich S, Chen CY, Menk J, Mueller B, et al. Transcranial direct current stimulation and constraint-induced therapy in cerebral palsy: A randomized, blinded, sham-controlled clinical trial. Eur J Paediatr Neurol. 2018;22:358-68. https://doi.org/10.1016/j.ejpn.2018.02.001 Elsevier Ltd.

21. Kirton A, Ciechanski P, Zewdie E, Andersen J, Nettel-Aguirre A, Carlson H, et al. Transcranial direct current stimulation for children with perinatal 
stroke and hemiparesis. Neurology. 2017;88:259-67 Available from: http:// cochranelibrary-wiley.com/o/cochrane/clcentral/articles/733/CN-01297733/ frame.html.

22. Moliadze V, Schmanke T, Andreas S, Lyzhko E, Freitag CM, Siniatchkin M. Stimulation intensities of transcranial direct current stimulation have to be adjusted in children and adolescents. Clin Neurophysiol. 2015;126:1392-9. https://doi.org/10.1016/j.clinph.2014.10.142 International Federation of Clinical Neurophysiology.

23. Grecco LAC, Duarte NAC, Zanon N, Galli M, Fregni F, Oliveira CS. Effect of a single session of transcranial direct-current stimulation on balance and spatiotemporal gait variables in children with cerebral palsy: a randomized sham-controlled study. Brazilian J Phys Ther. 2014;18:419-27 Available from: https://www.ncbi.nlm.nih.gov/pubmed/25372004.

24. Lazzari RD, Politti F, Santos CA, Dumont AJL, Rezende FL, Grecco LAC, et al. Effect of a single session of transcranial direct-current stimulation combined with virtual reality training on the balance of children with cerebral palsy: a randomized, controlled, double-blind trial. J Phys Ther Sci. 2015;27:763-8 Available from: https://www.jstage.jst.go.jp/article/jpts/ 27/3/27_jpts-2014-603/_article.

25. De Almeida Carvalho Duarte N, Grecco LAC, Galli M, Fregni F, Santos Oliveira C. Effect of transcranial direct-current stimulation combined with treadmill training on balance and functional performance in children with cerebral palsy: A double-blind randomized controlled trial. PLoS One. 2014;9 Available from: https://www.ncbi.nlm.nih.gov/pubmed/25171216.

26. Lazzari RD, Politti F, Belina SF, Collange Grecco LA, Santos CA, Dumont AJL, et al. Effect of transcranial direct current stimulation combined with virtual reality training on balance in children with cerebral palsy: a randomized, controlled, double-blind, clinical trial. J Mot Behav. 2017;49:329-36 Available from: https://www.ncbi.nlm.nih.gov/pubmed/27644454.

27. Grecco LAC, Oliveira CS, Galli M, Cosmo C, de Duarte N AC, Zanon N, et al. Spared primary motor cortex and the presence of MEP in cerebral palsy dictate the responsiveness to tDCS during Gait Training. Front Hum Neurosci. 2016;10:1-11 Available from: http://journal.frontiersin.org/Article/ 10.3389/fnhum.2016.00361/abstract.

28. Stinear CM, Barber PA, Smale PR, Coxon JP, Fleming MK, Byblow WD. Functional potential in chronic stroke patients depends on corticospinal tract integrity. Brain. 2007;130:170-80 Available from: https://www.ncbi.nlm. nih.gov/pubmed/17148468.

29. Kirton A. Advancing non-invasive neuromodulation clinical trials in children: lessons from perinatal stroke. Eur J Paediatr Neurol. 2017;21:75-103. https:// doi.org/10.1016/j.jpn.2016.07.002 Elsevier Ltd.

30. Staudt M. Reorganization after pre- and perinatal brain lesions. J Anat. 2010; 217:469-74 Available from: https://www.ncbi.nlm.nih.gov/pubmed/20649910

31. Mackey A, Stinear C, Stott S, Byblow WD. Upper limb function and cortical organization in youth with unilateral cerebral palsy. Front Neurol. 2014;5 JUL:1-9 Available from: https:/www.ncbi.nlm.nih.gov/pubmed/25071705.

32. Bradnam LV, Stinear CM, Barber PA, Byblow WD. Contralesional hemisphere control of the proximal paretic upper limb following stroke. Cereb Cortex. 2012;22:2662-71 Available from: https://www.ncbi.nlm.nih. gov/pubmed/22139791

Ready to submit your research? Choose BMC and benefit from:

- fast, convenient online submission

- thorough peer review by experienced researchers in your field

- rapid publication on acceptance

- support for research data, including large and complex data types

- gold Open Access which fosters wider collaboration and increased citations

- maximum visibility for your research: over $100 \mathrm{M}$ website views per year

At BMC, research is always in progress.

Learn more biomedcentral.com/submissions 